Note: This is a draft of a paper being submitted for publication. Contents of this paper should not be quoted nor referred to without permission of the authors.

Cunf-791112--54

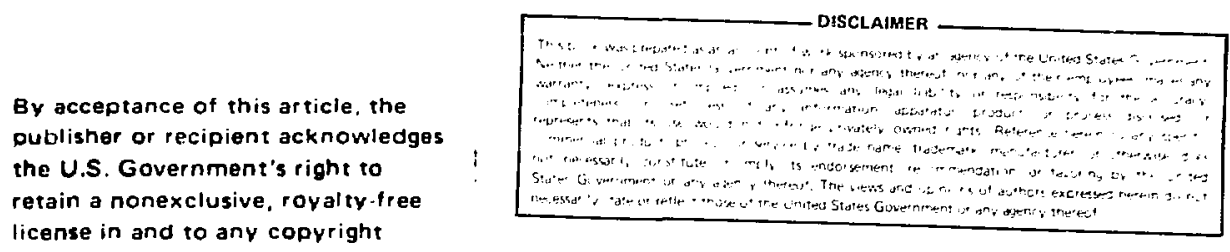
covering the article.

\title{
MACROSCOPIC THEOR'Y OF PULSED LASER ANNEALING
}

R. F. Wood, J. C. Wang, G. E. Giles and J.R. Kirkpatrick

SOLID STATE DIVISION

OAK RIDGE NATIONAL LABORATORY

Operated by

UNION CARBIDE CORPORATION

for the

U. S. Department of Energy

Oak Ridge, Tennessee

December 1979 
MACROSCOPIC THEORY OF PULSED LASER ANNEALING

\author{
R. F. Wood and J. C. Wang \\ Solid State Division, Oak Ridge National Laboratory \\ oak Ridge, Tennessee \\ G. E. Giles and J.R. Kirkpatrick \\ Computer Sciences Division, Oak Ridge National Laboratory \\ Oak Ridge, Tennessee
}

\begin{abstract}
Radiation from high-power Q-switched Zasers has been used recently in semiconductor research to anneal the lattice damage caused by ion implantation, diffuse surface-deposited dopant films, recrystallize doped amorphous films deposited on substrates, and remove precipitates present after conventional high-temperature dopant diffusion. All of these processes can be understood in terms of models based on macroscopic diffusion equations for heat and mass transport, cast in a finite-difference form to allow for the temperature- and spatial-iependence of the thermal conductivity, absorption coefficient, rejlectivity, and other quantities. Results of calculations on silicon with the models show that the near-surface region of a scomple can melt and stay molten for times of the order of 100 nsec during which dopant diffusion in the liquid state and nonequilibrium segregation during ultrarapid recrystallization are sufficient to explain the major features of the experimental results. In this paper, brief descriptions of the physical and mathematical models and some of the results obtained with them will be given, with particular emphasis on segregation effects.
\end{abstract}

\title{
I. INTRODUCTION
}

We restrict our attention here to the melting model of pulsed laser annealing because we believe the experimental evidence is overwhelmingly in favor of it. The reflectivity change exhibited by semiconductors during intense laser irradiation has been studied since about 1964. In some of the earliar papers, this reflectivity change was attributed to the high density of photogenerated carriers, i.e., to an electron-hole plasma. However, Blinov et al. (1) realized 
already in 1967 that this explanation did not fit their data on the absorptior, of long wavelength radiation during irradiation of Si and GaAs with a high-powered Q-Switched ruby laser. They concluded instead that the reflectivity change was due to the melting of a thin surface layer. A crucial question for the applicability of the melting model, at least in the form used here, concerns the lifetime of eiectron-hole pairs during intense laser irradiation and the transfer of energy from the electronic system to the lattice. There is a substantial body of literature on this topic and virtually all of the experimental data indicate that the electron-hole recombination time is between $.01 \mathrm{nsec}$ and $1 \mathrm{nsec}$. In fact, Svantesson et al. (2) found that in silicon the pulse width and shape of the recombination radiation in the region around $1.1 \mathrm{eV}$ (band gap of silicon) tracked the excitation pulse ( $30 \mathrm{nsec}$ ) almost identically. From the decay characteristics of the radiation, the authors concluded that the extremely fast recombination was due to Auger processes. In the earliest Soviet literature on laser annealing (3) it was recognized that melting of the near-surface region might explain the experimental results on dopant profile spreading. However, the results were not always consistent and this led some of the Soviet investigators (4) to assert that thermal models alone could not explain their results. These early experiments were done under coriditions which were not ideal and the dopant profiling was done almost exclusively by anodic oxidation and stripping rather than by Rutherford backscattering and SIMS (secondary ion mass spectroscopy) techniques. Furthermore, no detailed numerical calculations of temperature profiles, motion of the liquid-solid interface, and dopant redistribution were carried out. In fact, the calculations of Wang et al. (5) to explain experimental data obtained at Oak Ridge were the first to show convincingly that the near-surfase region could melt and that dopant diffusion in the molten state could explain the profile spreading which is observed. Similar calculations carried out independently by Baeri et al. (6) to explain their experimental data also appeared at approximately the same time and almost simultaneously experiments reported by Auston et al. (7) on the reflectivity change during laser annealing gave rather conclusive evidence that surface melting did occur. Since these experiments and calculations numerous other papers ha"e appeared which support the melting model. Of course, it is possible inspite of the experimental evidence to the contrary to maintain that melting does not occur, that the reflectivity change is due solely to the very high density of 1 aser-excited free carriers, and that some form of "radiation enhanced" diffusion is entirely responsible for the long-range diffusion of dopants. A theory 
along such 1 ines would have in the end to give almost identical results to the theory discussed here. Although it seems unlikely to us that such a theory can be constructed, it is certainly true that the effects of very high carrier densities need to be considered in the development of the melting model.

\section{HEAT TRANSFER CALCULATIONS}

\section{Heat Diffusion Equations}

We consider a sample irradiated with a single pulse of a Q-switched laser. The geometry of the sample and of the annealing configuration is such that ideally the heat conduction problem is well represented by the one-dimensiunal diffusion equation with a heat generation function determined by the interaction of the laser radiation and the sample. In simplest form for a sample in which the materials parameters are a constant, we can write the equation for the temperature $T(x, t)$ as

$$
\frac{\partial T(x, t)}{\partial t}-D_{t} \frac{\partial^{2} T(x, t)}{\partial x^{2}}=P(x, t),
$$

in which $P(x, t)$ is the heat generation function. $D_{t}$ is the thermal diffusion coefficient which involves the thermal conductivity, the specific heat and the density of the sample material. In laser annealing, the temperature of the sample is raised in a few nanoseconds to the melting point and even through the vaporization point if the laser pulse is sufficiently energetic. Both the thermal conductivity $k$, and the specific heat of $S i$ are highly temperature dependent (8). Equation 1 cannot describe such a situation and a more complex formulation of the problem based on finite differences is required. We found that a general purpose heat conduction computer program, HEATING5, (9) was easily adapted to laser annealing studies. This program solves steady-state and transient heat conduction problems in one, two, and three dimensions for cartesian, cylindrical, or spherical coordinates. The physical problem is approximated by a lattice of nodes, each associated with a small volume. A set of orthogonal planes defines the nodal system. Heat may flow between adjacent nodes along paths parallel to each coordinate axis. The system of equations describing the temperature distribution is derived from a heat balance condition at each node. For example, the finite-difference, heat-balance equation for node $i$ lying in the bulk of the sample is 


$$
c_{i} \frac{T_{i}^{n+1}-T_{i}^{n}}{\Delta t}=P_{i}^{n}+\sum_{m=1}^{6} i K_{m}^{n}\left(T_{m}^{n}-T_{i}^{n}\right)
$$

Here, $T_{m}^{n}$ is the temperature at node $m$ adjacent to node $i$ at time $t_{r} ;{ }_{j} K_{m}$ is the conductance between nodes $i$ and $m ; c_{j}$ is the heat capacitance of the material in the small volume around node $i$, and $P_{i}^{n}$ is the heat generation rate in this volume at time $\tau_{n}$. For a three-(one) dimensional problem, one $C$, one $P$, and six (two) $K$ 's may be associated with each internal node at a particular time, $t_{n}$. By choosing the increments between attice 1 ines and time steps small enough, the solution to tife system of equations yields an accurate approximation to the appropriate differential equation. Space- and time-dependent heat generation and temperaturedependent thermal properties are relatively easy to incorporate into finite difference calculations.

HEATING5 recognizes whether or not a node is undergoing a phase change. If it is, the node's temperature is maintained at the transition temperature until the net heat flow exceeds the node's latent heat of phase change. After the phase change, the node's temperature is again determined by the conductive heat transfer equation. The ratio of the node's heat energy above that required to just reach the transition temperature to the latent heat required for the phase change is called the transition ratio. The transition ratio can be used to locate the position of the melt front which is so important for laser annealing calculations.

Heat Generation Function, Reflectivity and Absorption Coefficient

In pulsed laser annealing, the reflectivity and absorption coefficient of the material and the energy density and pulse duration time of the laser pulse largely determine the heat generation rate at each point in the sample. The function $P(x, t)$ in Eq. 1 can be written as

$$
P(x, t)=(1-R) F(x, t),
$$

in which $R$ is the reflectivity and $F(x, t)$ can be a complex function of $x, t$, temperature and other materials parameters and physical phenomena. The reflectivity is also a complex function of a number of parameters describirig the near- 
surface region of the material. In most of our calculations on $\mathrm{Si}$, we have allowed $R$ to take on two values. One value describes the reflectivity of the surface before the laser pulse impinges on the sample and the other value gives the reflectivity after the near-surface region melts.

There are many discussions in the literature of the absorption mechanisms of intense laser radiation in solids. For our purposes the paper by Grinberg et al. (10) on the absorption of 1 aser radiation and the creation of damage by that absorption gives a good summary. Since this topic has al ready been discussed at length by others (see for example the paper by von Allmen in these Proceedings), we will not dwell on it here. In addition to those effects which occur even in perfect crystals, the ion-implantation process itself alters the absorption process. In undoped, single-crystal $\mathrm{Si}$, the absorption coefficient $k$ at a wavelength of $\lambda=0.694 \mu \mathrm{m}$ (ruby 1 aser) and at low light intensities is approximately $3 \times 10^{3} \mathrm{~cm}^{-1}$. Under the same conditions in amorphous $\mathrm{Si}, \mathrm{k}$ increases to a value of $\sim 5 \times 10^{4} \mathrm{~cm}^{-1}$. In ion-implanted $\mathrm{Si}$, the lattice damage created by implantation depends on the dopant, the implantation energy, and the dose. In some cases, such as $100 \mathrm{keV}$ implantation of arsenic, the near-surface region is driven almost completely amorphous. We may expect that for most implantation conditions and wavelengths of laser radiation the energy will be absorbed in both the damaged and the undamaged regions of the crystal. Finally, under intense laser irradiation it may happen that carrier diffusion effects can sometimes become significant (see the paper by $E$. Yoffa in these Proceedings, and these can be included approximately in terms of an effective absorption coefficient.

Definition of Two Models

Two basic models have been used in most of our heat conduction calculations. In one model the absorption coefficient is assumed to have some average, constant value throughout the sample. Such a model is suggested by a) ionimplantation of light ions such as boron in silicon where the implanted region is not made completely amorphous and the nature and extent of the damage is not very well defined; b) multipulse annealing where it is known that after the first laser pulse the damage in the implanted layer may be completely removed; and c) laser-induced melting of undoped, crystalline samples. Because of the absence of a welldefined damage layer in these cases we refer to this as the crystalline or c-model. In the second model a well-defined amorphous layer is assumed to be present. This model is expected to apply to the implantation of heavy ions into 
silicon in certain energy ranges and to samples on which an amorphous 1 ayer has been deposited by sputtering, e-beam deposition, etc. In this model the absorption coefficient has one value in the damaged region and some other value in the undamaged, crystalline region. We will refer to this model as the amorphous or a-model.

The latent heat of fusion is less for amorphous silicon than for single crystal silicon and presumatiy the melting points differ also. Reliable informatior about these quantities in ion-implanted materials is no', available. There are some indications that the latent heat of amorphous silicon may be roughly sixty-percent of the single crystal value and this has led to speculation that this would be an important effect in laser annealing. Our models have provisions for this difference in latent heat and we will give an example of its effect below.

\section{Some Results of Temperaturé Calculations}

The input data for these calculations consists of the thermal conductivity $K$, specific heat $c$, density $d$, reflectivity $R$, absorption coefficient $k$, latent heats of fusion (crystalline $L_{c}$ and amorphous $L_{a}$ ) and vaporization $L_{v}$, the corresponding temperatures $T_{C}, T_{a}$, and $T_{v}$ at which the phase changes occur, the pulse shape, duration $\tau_{\ell}$, and energy density $E_{\ell}$, and the parameters in the expressions for the radiative and convective heat transfer from the front surface. Most of these data are readily obtained from the literature and will not be discussed here.

An example of one of the most important results of the temperature calculations is given in Fig. 1. This figure is

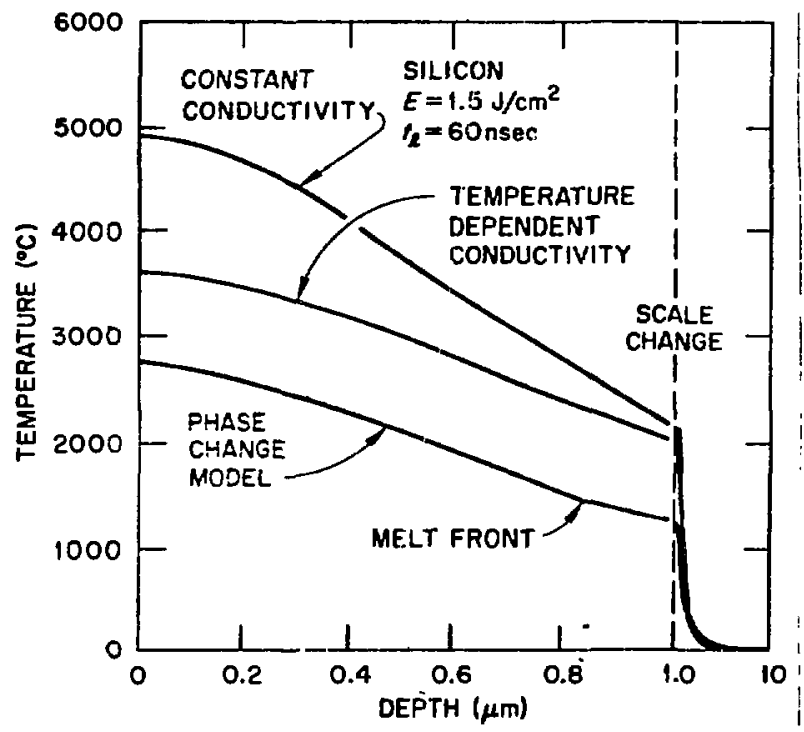

FIGURE 1. Temperature as a function of depth for a number of approximations discussed in the text. In this figure $E$ is the energy density and $t_{l}$ the duration of the incident laser pulse. 
based on very early calculations which did not contain a reflectivity switch on melting; it is used here for illustrative purposes only. The top curve is a typical temperature distribution obtained from $\mathrm{Eq}$. 1 when melting is not allowed and $K$ and $C$ are assumed to be constant with temperature. The middle curve shows the effects of including the temperature dependence of $K$ and $c$. The calculations leading to the lowest curve allowed for temperature-dependent $K$ and $c$ and for melting. This figure shows the importance of allowing for these effects in the calculations.

From a series of calculations such as those leading to the lowest curve on Fig. 1 the melt-front position as a function of time can be obtained. Examples of such curves are shown in Fig. 2 for a $25 \mathrm{nsec}$ laser pulse of various energies. Note that these calculations included a reflectivity switch from $R_{S}=0.35$ (solid) to $R_{m}=0.60$ (molten) on melting. The absorption coefficient is assumed to have an average value of $3 \times 10^{4} \mathrm{~cm}^{-1}$ throughout the materia?. From the curves on Fig. 2 it is found that the recrysti.11ization velocity is of the order of $3-4 \mathrm{~m} / \mathrm{sec}$. Figures 1 and 2 provide the key to understanding the details of pulsed-laser annealing. Much of our work has been directed toward obtaining melt-front histories such as those contained in Fig. 2 for a variety of assumptions about the thermal conductivity, absorption coefficient, reflectivity, pulse duration time, etc. A more detailed account of this work will be given in a later publication (11).

In Fig. 3, we show the effects of reducing the heat of fusion in the amorphous 1 iyers, i.e., of taking $L_{a}<L_{c}$. The basic model for these calculations had $R_{S}=0.35, R_{\mathrm{m}}=0.60$, $x_{a}=0.15 \mu \mathrm{m}$ (thickness of amorphous layer), $k_{a}=5 \times 10^{4} \mathrm{~cm}^{-1}$,

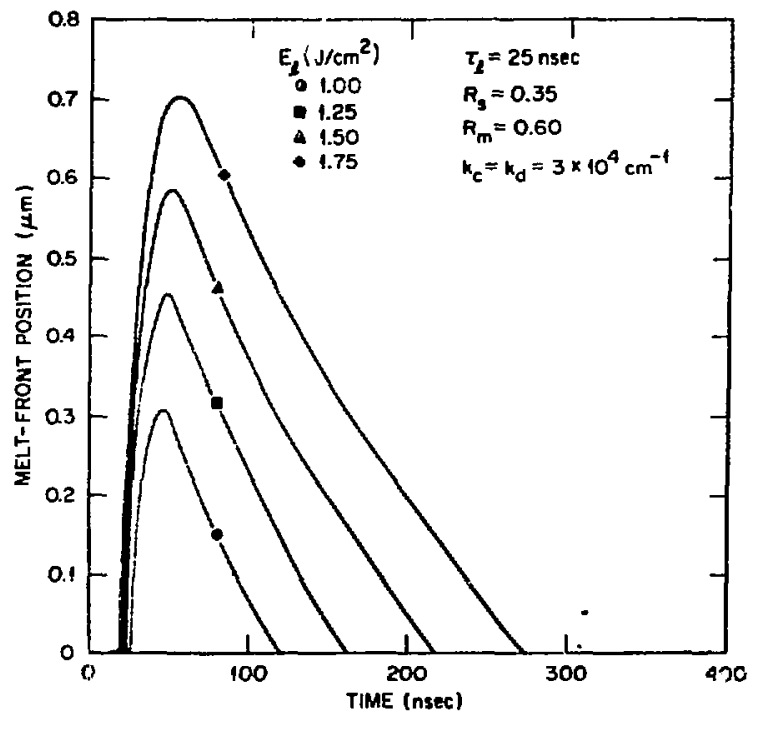

FIGURE 2 An example of the results of melt-front profile calculations. 
and $k_{c}=3 \times 10^{3} \mathrm{~cm}^{-1} ; k_{a}$ and $k_{c}$ are the absorption coefficients in the amorphous and crystalline regions respectively. Curve a gives the melt-front history for a laser pul se of $E_{\ell}=1.4 \mathrm{~J} / \mathrm{cm}^{2}, \tau_{\ell}=15 \mathrm{nsec}$, and $\mathrm{L}_{a}=\mathrm{L}_{c}$ and curve $b$ is for the same conditions but with $\mathrm{L}_{a}=0.6 \mathrm{~L}_{c}$. Curves $c$ and $d$ are for the same sequence of calculations but with $E_{\ell}=1.5 \mathrm{~J} / \mathrm{cm}^{2}$ and $\tau_{\ell}=60$ nsec. Clearly, a substantial reduction in the latent heat does not cause very large changes in the melt-front histories, as has been suggested by others. The reasons for this somewhat surprising result are quite simple and are directly related to the role played by the reflectivity switch. It should be understood that this result applies only to the conditions discussed here. The difference between $L_{a}$ and $L_{c}$ plays a much greater role in annealing with $\mathrm{CW}$ lasers where recrystallization occurs by solid-phase epitaxy.

Figure 4 is our final example of the results of heat conduction calculations; it shows the time the surface remains molten as a function of incident laser energy. The experimental data is from Auston et al. (7). The solid squares and circles give the results of calculations using sets of parameters which differ only slightly from one another. In fact, the differences are too slight to warrant discussion here. The open circles and squares are calculations more appropriate to annealing with a ruby laser $(\lambda=0.694 \mu \mathrm{m})$ and are based on an amorphous model with $X_{a}=0.15 \mu \mathrm{m}$ and two different values of $k_{a}$. These and other calculations which we

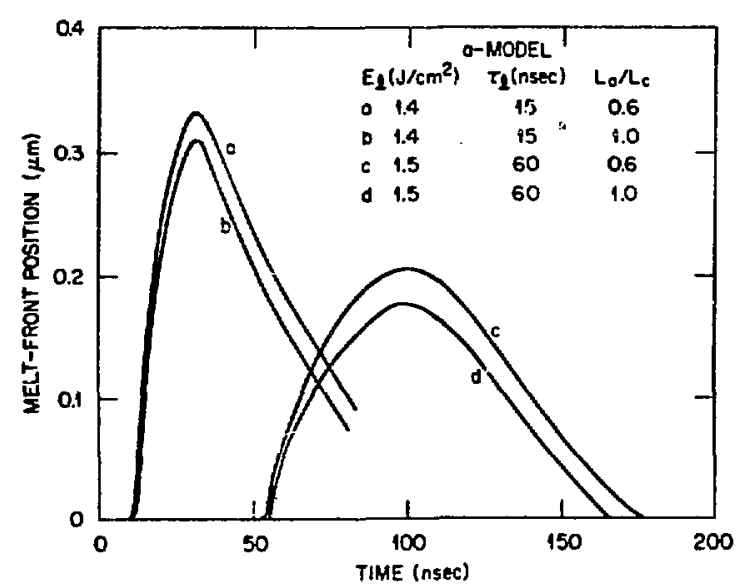

FIGURE 3. Mlustrations of the effects of a latent. heat reduction in the amorphous layer on melt-front profiles.

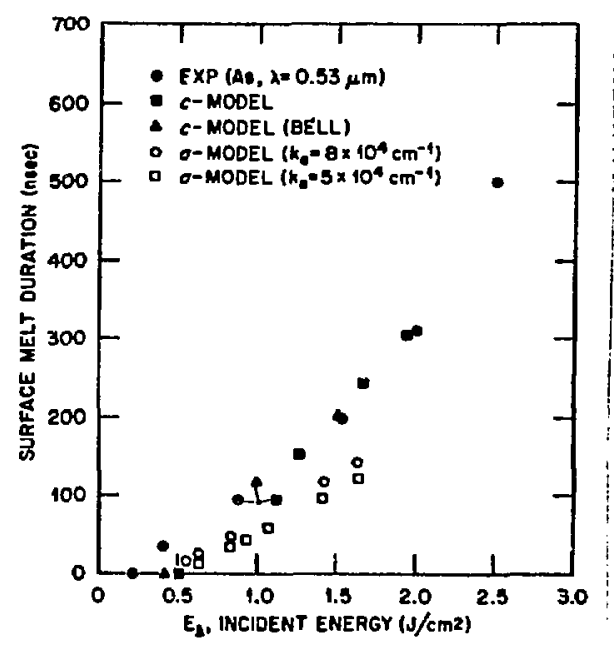

FIGURE 4. Surface melt duration as a function of incident energy for a number of different models. 
have made show that the surface meit duration depends strongly on a number of conditions under which the laser annealing is carried out. Experimental data on the duration of surface melting is very useful as an aid in determining some of the parameters in a given model but such data alone cannot uniquely determine the model to the extent that the melt-front profile, penetration, and velocities can be predicted. The fitting of experimental dopant profiles with a model for diffusion in the liquid state is a great aid in the overall development of the theory of pulsed laser annealing and we now consider a number of aspects of the dopant diffusion problem.

\section{DOPANT DIFFUSION WITHOUT SEGREGATION}

As we have shown above, during pulsed laser annealing the near-surface region of an ion-implanted sample melts. While this region is molten, rapid diffusion of the dopant occurs (5). In those cases where the melt front penetrates well beyond the implanted dopant profile, rather good fits to the experimental data can be obtained by assuming that the region of the profile is instantaneously melted, stays molten for a certain period of time, and then is instantaneously resolidified. This "instantaneous approximation" (IA) fails when the maximum melt-front penetration occurs within or just beyond the implanted profile and improved approximations in which the actual motion of the melt front is inciuded must be constructed. We have employed two types of approximations to deal with this moving boundary value problem.

The first approximation is derived from the Green's function formulation of the one-dimensional, mass-diffusion problem. In the IA, the profile after laser annealing is given by

$$
\rho(x, t)=\int_{0}^{\infty}\left[G\left(x t \mid x_{0} t_{0}\right) \rho_{j}\left(x_{0}, t_{0}\right)\right]_{\vec{t}_{0}=0} d x_{0}
$$

in which $\rho_{i}$ and $\rho$ are the implanted and final profiles respectively and $G\left(x t \mid x_{0} t_{0}\right)$ is the Green's function for diffusion in a semi-infinite sample. For assumed values of the diffusion parameters, a fit of $\rho$ to the experimental profile determines the time during which the implanted region remained molten. This time should be consistent with that obtained from the calculations of temperature and melt-front motion such as those described in the preceding section. obviously, when the melt-front does not penetrate beyond the 
implanted profile, a single time during which the entire profile is allowed to diffuse cannot be assigned in even an approximately correct manner. A simple approximation which gives surprisingly good results and yet remains within the spirit of $\mathrm{Eq} .4$ is obtained from the following procedure. From plots of the melt-front location as a function of time such as those given in Fig. 2, the time during which any point in the sample remains molten can be determined. Substituting these times into a suitably modified form of $\mathrm{Eq} .4$ and carrying out the integration over $x_{0}$ gives an approximation to the diffused profile.

Because the approximations on which the above-described procedure is based are difficult to justify and may not always apply, we have used another model based on a finitedifference formulation of the mass-diffusion equation

$$
\frac{\partial \rho(x, t)}{\partial t}=\frac{\partial}{\partial x}\left[D \frac{\partial \rho(x, t)}{\partial x}\right] \text {, }
$$

with appropriate boundary conditions. The diffusivity $D$ can be a function of $x$ and $t$ and this flexibility is used to change from values characteristic of the 1 iquid $\left(D_{\ell}\right)$ to those characteristic of the solid $\left(D_{S}\right)$ at the moving liquid-solid interface. The sample is imagined to be oriented perpendicular to the $x$ axis with its front face at $x=0$ and its 0 thickness divided into cells with $\Delta x$ of the order of $100 \mathrm{~A}$ or smaller. As the melt front penetrates the sample and moves through a cell, the value of $D$ in the cell changes from $D_{S}$ to $D_{\ell}$. The material in all cells to the left of the melt front is molten and diffusion occurs very rapidly within it. When the melt front reaches its maximum penetration and begins to recede back to the surface, solidification occurs and the value of $D$ in each cell is changed accordingly. The interface segregation :oefficient $k_{i}$ determines the fraction of the dopant which is incorporated into the solid. The dopant rejected into the liquid at the interface combines with the implanted dopant in the molten zone to give $\rho(x, t)$ of $\mathrm{Eq}$. 5. The numerical methods used in the dopant diffusion problem are similar to though not as complex as those used in HEATING5. Brief discussions of the details of dopant diffusion calculations can be found in several other papers in these Proceedings and we will not pursue this topic further here.

Figure 5 shows the results of dopant profile calculations on As-implanted Si using the approximate Green's function method described above. The experimental profiles were measured by ion backscattering and with the possible exceptions of a very few points, the calculated curves fall within 


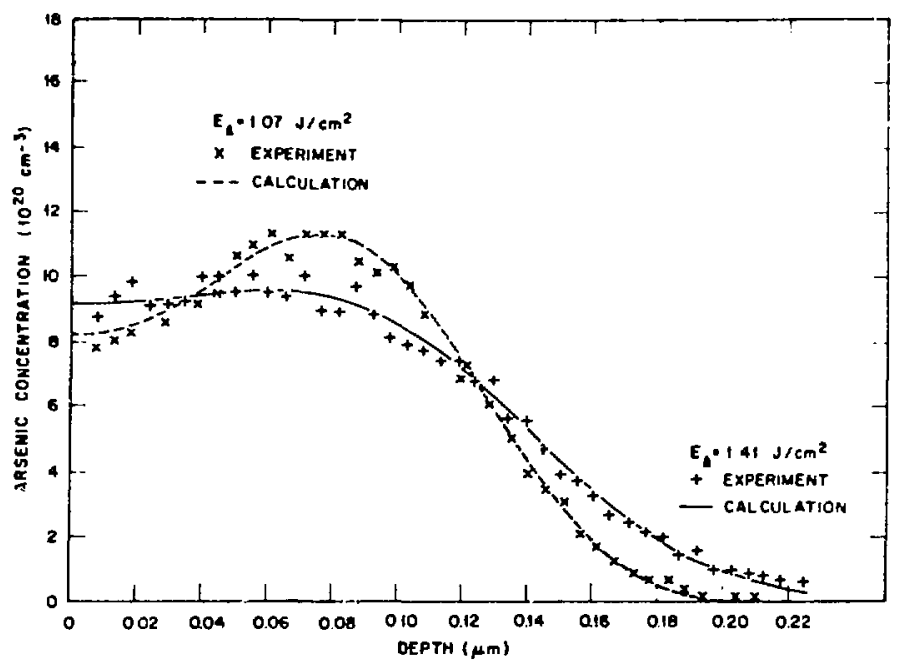

FIGURE 5

Experimental and calculated dopant profizes in Asimpionted, Zaseranneaied sizicon.

the estimated error limits of the experiments. Dopant profiles obtained when As-doped amorphous 1 ayers of $\mathrm{Si}$ are deposited on Si substrates and then recrystallized by laserinduced melting are shown in Fig. 6. The experimental profiles in this case were determined by anodic oxidation and stripping. The starting profiles cannot be measured by this techrique because most of the As is not electrically active when the deposited layer is still amcrphous. However, the deposition is such that the As concentration in the deposited layer should be nearly uniform. The uniform concentrations required to give the calculated piofiles are shown by the dashed lines in the figure. The value of $D_{\ell}$ for As in Si was taken from Koderà (12).

The dopant profiles in Figs. 5 and 6 were calculated with $k_{i}=1$ whereas the compilation of Trumbore (13) gives a value of 0.3 for the equilibrium interface segregation coefficient of As in Si. We now turn to a consideration of this discrepancy.

\section{NONEQUILIBRIUM SEGREGATION}

In one of the earliest papers on laser annealing of ionimplanted Si, Khaibullin et al. (3) reported that the concentration of dopants after annealing could exceed conventional solubility limits. They recognized that this indicated that nonequilibrium thermodynamic processes occur during iaser annealing. As we have seen in Section II, calculations have shown that the near-surface regions of the sampies meit and recrystallize in times of the order of 100 nsec and that the velocity $v$ of the liquid-solid interface during recrystallization is of the order of $4 \mathrm{~m} / \mathrm{sec}$. Nonequilibrium segregation effects during rapid recrystallization have been discussed in the literature on crystal growth (14) but, until the discovery of the laser-annealing 
phenomenon, the melt-front velocities attainable under wellcontrolled experimental conditions were too small to test various proposed models, most of which can now be shown to be inadequate or incomplete. In this Section, a simple phenomenological model which does account for the observed segregation effects is presented (15).

The interface segregation coefficient $k_{j}$ for the dopant is defined as the fraction of the dopant present in the liquid at the interface that is incorporated into the solid. If equilibrium values $k_{j}^{0}$ were followed in the laser annealing process, then large concentration spikes at the surface would be observed for most dopants implanted in Si. Such surface spikes have not been observed for $B$, As and $P(16,17)$ and satisfactory fits to the profiles can only be obtained with values of $k_{j}$ nearly equal to unity (see for example Figs. 5 and 6). The fact that equilibrium solubility limits can be exceeded in systems such as these, which have retrograde solubility, can be taken as further evidence for nonequilibrium growth processes $(3,14,18,19)$.

Recently White et al. (19) used RBS to measure the profiles of $\mathrm{Ga}$, In and $\mathrm{Bi}$ in ion-implanted, laser-annealed Si. The results, together with earlier data on Sb and As were analyzed using a model of dopant diffusion which included segregation effects and utilized melt-front information from

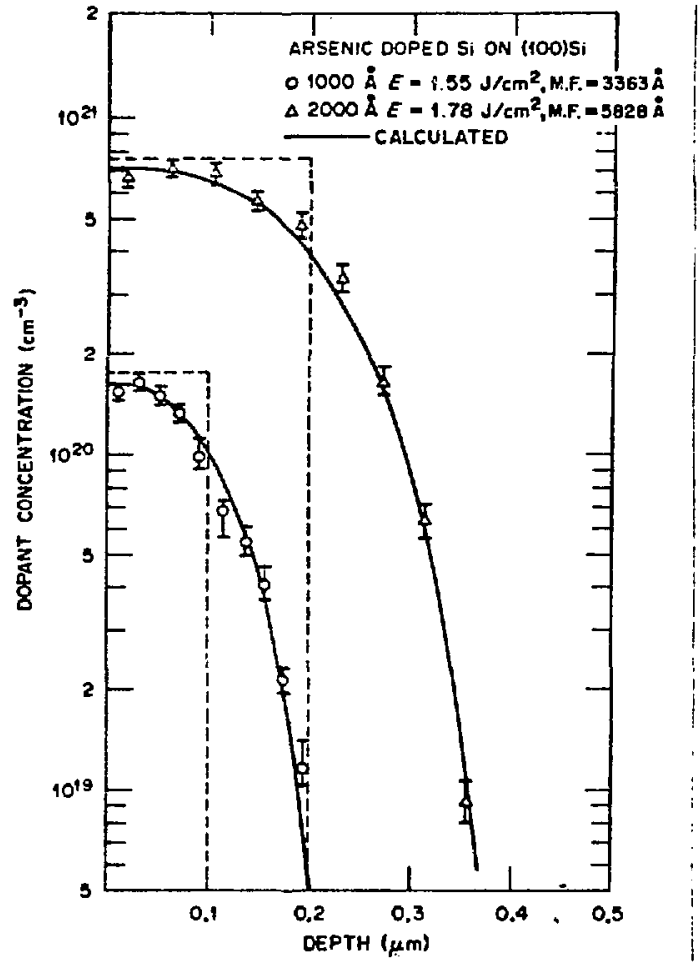

FIGURE 6. Experimental and caloulated dopant profiles after laser-induced recrystallization of $1000 \AA$ and $2000 \AA$ Layers of Asdoped amorphous $S i$ deposited on Si. In this figure $E$ gives the incident Laser energy density and M.F. the calculated maximum melt-front penetration. 
calculations such as those in Section II; from fits of the measured profiles, values of $k_{i}$ were extracted. The results of White et al. and of refs. 5,16 , and 17 provide data on the segregation behavior of $B, P, A s, S b, G a$, In and $B i$ in $S i$ during laser annealing. Values of $k_{j}^{O}$ and $k_{j}$ are shown in Table I. The most striking feature of these data is the very large differences between $k_{j}^{0}$ and $k_{j}$ when $k_{i}^{O}$ is small.

The model to be described now $1 \mathrm{~s}$ based on the assumption that for $v \gg 0$ new layers of atoms may be added to the solid so quickly that dopant atoms in the interface region have a reduced probability of escape from the solid being formed. Conceptually the model is related to what Baker and Cahn (14) have referred to as "solute trapping".

The rate equations for the incorporation of host $h$ and dopant $d$ atoms into the solid are (20)

$$
R_{j}^{S}=K_{j}^{f} C_{j}^{i}-K_{j}^{r} C_{j}^{S} \quad, j=h, d,
$$

where $c_{j}^{L}$ and $c_{j}^{S}$ are the concentrations of $j$-type atoms in the liquid and solid, and $\mathrm{K}_{\mathrm{f}}^{\mathrm{f}}$ and $\mathrm{K}_{\mathrm{r}}^{\mathrm{r}}$ are the forward (liquid $\rightarrow$ solid) and reverse rate constants in $\mathrm{m} / \mathrm{sec}$. Since the crystal grows at a rate equal to the melt front velocity, addition of the individual rate equations gives

$$
v=R_{h}^{S}+R_{d}^{S}=K_{h}^{f} C_{h}^{L}+k_{d}^{f} c_{d}^{L}-\left(K_{h}^{r} C_{h}^{S}+K_{d}^{r} C_{d}^{S}\right)
$$

with

$$
c_{h}^{S}+c_{d}^{S}=c_{h}^{L}+c_{d}^{L}=1
$$

It will be assumed that $K_{h}^{f}=K_{d}^{f}=K_{h}^{f o}=K_{d}^{f o}$, where $K_{j}^{f o}$ is the forward rate constant under equilibrium growth conditions $(v \simeq 0)$; this is not a crucial assumption but it simpl ifies the analysis. With this assumption, Eq. (6) for $R_{d}^{S}=v C_{d}^{S}$ and

\begin{tabular}{|c|c|c|c|c|c|c|}
\hline Dopant & $k_{i}^{0}$ & $\begin{array}{c}k_{i} \\
(\exp )\end{array}$ & $\begin{array}{c}k_{i} \\
v_{0}=c o n .\end{array}$ & $v_{0}=D_{\ell}^{k_{i} / x_{0}}$ & $\begin{array}{c}\Delta U_{d}^{r a} \\
e V\end{array}$ & $c_{d}^{S} / C_{d}^{S O}$ \\
\hline $\bar{B}$ & 0.8 & 1.00 & 0.98 & 0.99 & -0.032 & -- \\
\hline$P$ & 0.35 & 1.00 & 0.90 & 0.95 & -0.152 & 1.5 \\
\hline As & 0.3 & 1.00 & 0.88 & 0.97 & -0.175 & 1.4 \\
\hline$S o$ & 0.023 & 0.7 & 0.68 & 0.88 & -0.547 & 26 \\
\hline$G z$ & 0.008 & 0.2 & 0.61 & 0.31 & -0.700 & 14 \\
\hline$I r_{l}$ & 0.0004 & 0.15 & $0: 45$ & 0.15 & -1.134 & 400 \\
\hline$B i$ & 0.0007 & 0.4 & $0: 48$ & 0.39 & -1.053 & 1000 \\
\hline$A Z$ & 0.0020 & - & 0.53 & 0.52 & -0.901 & 55 \\
\hline
\end{tabular}

Table I. Results of calculation of $k_{i}$ and comparison to experiment. In the fourth column, con. $=1.75 \mathrm{~m} / \mathrm{sec}$. 
Eq. (7) yield

$$
K_{d}^{f o} C_{d}^{S}-\left(K_{h}^{r}-K_{d}^{r}\right) C_{d}^{S}\left(1-C_{d}^{S}\right)=K_{d}^{f o} C_{d}^{L} \text {. }
$$

Under most conditions of laser annealing $C_{d}^{S}$ is not more than a few percent and therefore to the accuracy needed here $\left(1-C_{d}^{S}\right) \simeq 1$. The interface segregation coefficient for the dopant, $k_{j}=C_{d} / C_{d}^{L}$, can then be expressed as

$$
k_{i}=k_{d}^{f o} /\left(k_{d}^{f o}-k_{h}^{r}+k_{d}^{r}\right) \text {. }
$$

We assume that the rate constant $k_{d}^{r}$ is of the usual form

$$
K_{d}^{r}=A_{d}^{r} \exp \left(-U_{d}^{r} / R T\right) \text {, }
$$

in which $U_{d}^{r}$ is the barrier height for escape of dopant atoms from the solid at the interface; similar forms are assumed for $K_{h}^{\text {fo }}$ and $K_{h}^{r}$. Furthẹrmore, $U_{d}^{r}$ is assumed to be velocity dependent and written as

$$
u_{d}^{r}(v)=u_{d}^{r o}+\Delta u_{d}^{r}(v) \text {, }
$$

where $u_{i}^{r o}$ is the equilibrium value. Now $\Delta u_{d}^{r}$ nay be a complicated function of $v$, but it must approach zero as $v \rightarrow 0$ and a constant value as $v$ becomes large. The simple form

$$
\Delta u_{d}^{r}(v)=\Delta u_{d}^{r a}\left(1-\exp \left(-v / v_{0}\right)\right)
$$

satisfies these conditions; $\Delta U_{d}^{r a}$ is the asymptotic value of $\Delta u r$ as $v$ becomes very 7 arge and $v_{0}$ is a parameter discussed below.

$$
K_{d}^{r}=F K_{d}^{r o} \exp \left(-\Delta U_{d}^{r}(v) / R T\right)
$$

with $F \equiv r_{d} / A_{d}^{r o}$.

Since both $c_{d}^{L}$ and $c_{d}^{S}$ are small, $c_{h}^{L} \simeq C_{h}^{S}$, and from Eq. (6) $v=K_{h}^{f o}-K_{h}^{r}$ because of the simplifying assumption that $K_{h}^{f}=K_{h}^{f o}$. Rate constants have been estimated to be of the order of $100 \mathrm{~m} / \mathrm{sec}(21)$. Assuming this value to be approximately vaitd for $S i, K_{h}^{r}$ must be $96 \mathrm{~m} / \mathrm{sec}$ to obtain $v=4$ $\mathrm{m} / \mathrm{sec}$. The maximum value $k_{j}$ can have for the systems of interest here is 1 and, with the previous assumptions that $K_{d}^{f o}=K_{h}^{f}$, this occurs when $K_{d}^{r}=K_{h}^{r}$. Hence, in Eq. (10) the term $K_{d}^{f o}-K_{h}^{r}$ compared to $K_{d}^{r}$ can be neglected. With the definition $k_{i}^{0} \equiv\left(C_{d}^{S} / C_{d}^{L}\right)_{v=0}=K_{d}^{f o} / K_{d}^{r o}$, Eq. (10) becomes 


$$
k_{i}=\left(k_{i}^{0} / F\right) \exp \left(\Delta U_{d}^{r}(v) / R T\right) .
$$

From the requirements that $k_{j} \rightarrow k_{i}^{0}$ as $v \rightarrow 0$ and $k_{j} \rightarrow 1$ as $v$ becomes large, it is found that $F=1$,

$$
\Delta U_{d}^{r a}=-R T \ln k_{i}^{0},
$$

and hence

$$
k_{i}=k_{i}^{0} \exp \left(\left(-R T 1 \| k_{j}^{0}\right)\left(1-\exp \left(-v / v_{0}\right)\right) / R T\right) .
$$

Column 4 of Table I shows the results when a single value of $v_{0}$ is used in Eq. (7). Clearly, the model can account for the extraordinarily large differences observed between $k_{j}^{0}$ and $k_{i}$. There is no reason $v_{0}$ should be the same for all dopants and experimentation suggested that the diffusion coefficient in the liquid $D_{\ell}$, was a relevant parameter. Putting $v_{0}=$ $D_{\ell} / x_{0}$ and $x_{0}=225 \AA$ gave the results in column 5 . Values of $D_{\text {\& }}$ were taken from Kodera (12) and varied within his stat d $_{\text {error }}$ ranges to give the fits to $k_{j}$ (exp) in Table $I$. Only for SD and Ga are there appreciable differences between the experimental and calculated values. Approximately $10 \%$ and $40 \%$ respectively of these dopants were lost during annealing and this made a unique fit to the experimental profiles difficult. Thus, the overall fit ic remarkably good. With $\mathrm{Eq} .(17), k_{j}$ was calculated as a function of $v$ and the results are given in Fig. 7 .

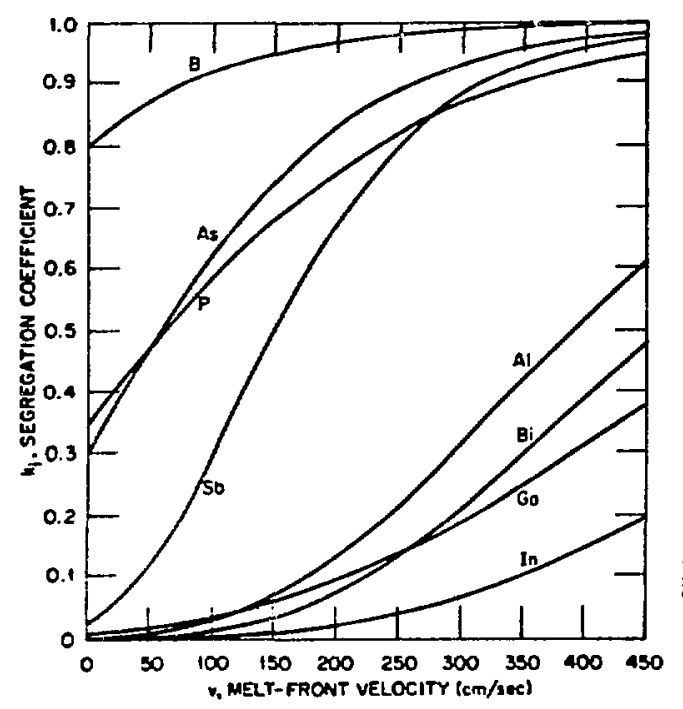

FIGURE 7. Dependence of $k_{i}$ on melt-front velocity. 
The equation

$$
v=K_{h}^{f}\left(\Delta H_{h} / R T_{M}^{2}\right) \Delta T \quad, \Delta T \equiv\left(T_{m}-T\right)
$$

can be derived (see e.g. Thurmond, ref. 20) from Eq. 6. $\Delta H_{h}$ and $T_{M}$ are the heat of fusion and the melting temperature respectively of the host. $\Delta T$, the temperature drop across the interface which drives the melt front can not be discontinuous and must have a distance $\Delta x$ associated with it. From the calculations of heat transport such as those in Section II that determined $v,(\partial T / \partial x)$ at the melt front can also be determined and for the conditions of the present experiments, it is $\approx 10^{7} \mathrm{o} / \mathrm{cm}$. Using $v=4 \mathrm{~m} / \mathrm{sec}, \mathrm{Kh}_{\mathrm{h}} \simeq 100 \mathrm{~m} / \mathrm{sec}, \Delta H_{\mathrm{h}}=$ $12 \mathrm{kcal} / \mathrm{mgle}$ and $T_{M}=1683^{\circ} \mathrm{K}$, it is found that $\Delta T \simeq 20^{\circ} \mathrm{K}$ and $\Delta x=200 \AA$. This calculation suggests an obvious interpretation of $x_{0}$ in $v_{0}=D_{\ell} / x_{0}$, i.e., it can be equated with $\Delta x$ and referred to as the width of the interface region. In the interiace region, dopant diffusion is characterized by a diffusion coefficient $D_{i}$ which is of the order of $D_{l}$. The undercooling or supercooling given by $\Delta T$ does not dejend on the dopant concentration; it is a property of pure Si. In addition, at high doping concentrations in the interface region, constitutional supercooling can be expected and may lead to the formation of cellular structures which have been observed in some cases during laser annealing.

Coiumn 6 in Table I gives values of $\Delta u_{d}^{r a}$. When these "trappiag energies" are compared to the activation energy $E_{a}$ for diffusion in the solid (22) it is found that $\Delta U_{d}^{\text {ra }}$ is a rather small fraction of $E_{a}$ for the dopants 1 isted in Table I. Thus, it is suggested that the bonding between the dopant and host atoms is easily great enough to account for the trapping. The situation is quite different for dopants such as $\mathrm{Cu}$ and $\mathrm{Fe}$. Values of $k_{i}^{0}$ (ref. 13) are $4 \times 10^{-4}$ and $8 \times 10^{6}$ respectively and the corresponding values of $E_{a}$ are $\sim 1$ $\mathrm{eV}$ and $0.87 \mathrm{eV}$ whereas, from Eq. (16), $\Delta \mathrm{Ud}_{\mathrm{d}}^{\mathrm{ra}}$ for $\mathrm{Cu}$ is -1.13 $\mathrm{eV}$ and for $\mathrm{Fe}$ it is $-1.70 \mathrm{eV}$. The bonding properties of $\mathrm{Fe}$ and $\mathrm{Cu}$ in $\mathrm{Si}$ are not sufficient to provide the necessary trapping energy.

From the relationship $c_{d}^{L}=1-c_{h}^{L}$ and the expression for $c_{h}$ in an ideal dilite solution (e.g., Thurmond $p .149$, ref. 20), the equation

$$
c_{d}^{S}=k_{i}\left(1-\exp \left(-\Delta H_{h} \Delta T / R T_{M}^{2}\right)\right)
$$

can be obtained. With the values of $k_{j}$ in column 5 of Table $I$ and $\Delta T=20^{\circ} \mathrm{K}, C_{d}^{S}$ was calculated. The ratio of $C_{d}^{S}$ to the maximum equilibrium solubility $C_{d}^{S o}$ extracted from ref. 13 
are given in the last column. These ratios are in quite satisfactory agreement with the experimental values given in ref. 19 and in the paper by C.W. White, S. R. Wilson, and B. R. Appleton in these Proceedings.

\section{ACKNOWLEDGMENTS}

We would like to thank M. Mostoller, T. Kaplan, M. Rasolt, and $F$. W. Yourig, Jr. for useful discussions and the authors of reference 19 for making their data available to us before publication. This research was sponsored by the Division of Materials Sciences, U. S. Department of Energy under contract W-7405-eng-26 with the Union Carbide Corporation.

\section{REFERENCES}

1. L. M. Blinov, V. S. Vavilov, and G. N. Galkin, Sov. Phys. Semiconductors 1, 1124 (1967).

2. K. G. Svantesson, $\mathrm{N}_{\mathrm{k}}$ G. Nilsson, and L. Huldt, Sol. St ate Comm. 9, 213 (1971).

3. I. B. Khaibullin, E. I. Shtyrkov, M. M. Zaripov, M. F. Galyautdinov, and G. G. Zakirov, Sov. Phys. Semicond. 11,190 (1977).

4. I. B. Kisaibullin, E. I. Shtyrkov, M. M. Zaripov, R. M. Bayazitov, and M. F. Galjautdinov, Radiat. Eff. 36, 225 (1978).

5. J. C. Wang, R. F. Wood, and P. P. Pronko, Appl. Phys. Lett. 33, 455 (1978).

6. P. Baeri, S. U. Campisano, G. Foti, and E. Rimini, Appl. Phys. Lett. 33, 137 (1978).

7. D. H. Auston, C.M. Surko, T.N.C. Venkatesan, R. E. Slusher, and J. A. Golovchenko, Appl. Phys. Lett. 33, 437 (1978).

8. H. R. Shanks, P. D. Maycock, P. H. Sidles, and G. C. Danielson, Phys. Rev. 130, 1743 (1963).

9. The HEATING5 program has been developed over $;$ period of years at the Oak Ridge National Laboratory. It is probably quite similar to large heat conduction programs developed at a number of other 1aboratories.

10. A. A. Grinberg, R. F. Mekhtiev, S. M. Ryvkin, V. M. Salmanov, and I. D. Yaroshetskii, Sov. Phys.-Solid State 9, 1085 (1967).

11. R. F. Wood and G. E. Gi ?es (to be submitted to Phys. Rev.).

12. H. Kodera, J. Appl. Phys. (Japan) 2, 212 (1963).

13. F. A. Trumbore, 'Bell 'jystem Tech. J. 39, 205 (1960).

14. J. C. Baker and J. W. Cahn, Acta Met. 17, 575 (1969).

15. This section is taken in large part from a paper recently submitted for publication by one of us (RFW). 
16. C. W. White, J. Narayan, and R. T. Young, Science 204, 461 (1979).

17. R. T. Young, J. Narayan, and R. F. Wood, Appl. Phys. Lett. 35, 447 (1979).

18. K. A. Jackson and H. J. Leamy, Laser-Solid Interactions and Laser Processing, ed. by $H . J$. Leamy and J. M. Poate (American Institute of Physics, New York, 1979), p. 102.

19. C. W. White, S. R. Wilson, and B. R. Appleton, J. Appl . Phys. (to be published) .

20. See for example, K. A. Jackson, Can. J. Phys, 36,683 (1958), and C. D. Thurmond in Semi conductors, ed. by N. B. Hannay (Reinhold, 1959), p. 145.

21. B. Chalmers, Trans. AIME 200, 519 (1954).

22. B. L. Sharma, Diffusion in Semi conductors, (Trans. Tech. Publications, Claustha1-Zellerfeld, Germany, 1970). 